\title{
Chain-based Gateway nodes routing for energy efficiency in WSN
}

\author{
Amir Hozhabri*1, Mohammadreza Eslaminejad ${ }^{2}$, Mitra Mahrouyan ${ }^{3}$ \\ 1 *- Department of Computer Science and Information Technology, \\ Zand Institute of Higher Education, Shiraz, Iran \\ 2- Department of Computer Science and Information Technology, \\ Zand Institute of Higher Education, Shiraz, Iran \\ 3- Department of Physics, Faculty of Science, University of Isfahan, Isfahan, Iran
}

\begin{abstract}
Energy storage is the dominating factor in Wireless Sensor Networks (WSN). It is important to choose a routing strategy for the appropriate delivery of packages. This research provided an analysis of energy-efficient chain-based routing, focusing on routing methods in WSN and analyzing previous methods. The inspiration came from gateway nodes instead of chain head nodes and a new method was discussed to improve energy efficiency and the lifespan of networks. In the proposed methods, the gateway nodes were provided with rechargeable solar batteries. Moreover, there was a gateway node in each chain for collecting data and directly sending it to the sink node or Base Station (BS). Therefore, these methods could reduce energy consumption as well as the end-to-end and overall delay of network and increased the network lifespan, compared to previous methods. The simulation results superlatively showed that in the new algorithm, the energy in the nodes was conserved more than $50 \%$ and the networks' lifespan was prolonged at least $\mathbf{4 6 . 4 2 \%}$ more than previous methods.
\end{abstract}

Keywords: Routing in Wireless Sensor Network, Chain-based routing, Energy-storage, Gateway node

\section{Introduction}

WSN is a wireless computer network technology that consists of many other systems, equipped with sensors. In the past, the size of nodes was bigger, but nowadays thanks to the progress in electronics fields, they are smaller; therefore, this network is more efficient. The wireless sensor nodes receive their required energy from the batteries, so the nodes' energy consumption should be controlled in order to prolong their and the whole network's lifespan. In WSN, the highest rate of energy consumption is in communication fields.

WSN consists of many sensors that are densely planted in one place to sense, collect, process, and send data wirelessly (Warrier. 2016; Akyildiz, 2002; Salahuddin, 2015; Vahabi, 2019). Each wireless sensor is called a wireless sensor node (WSN). WSN energy is limited by batteries. WSN is small and smart and costs-effective (Salahuddin, 2015). These networks can be applied for dangerous places, supervising an area, and automation (Vahabi, 2019) and have many military and non-military applications including tracing, agricultural and animal husbandry programs, supervision over patients in medical centers, etc.

WSN routing protocols are divided into three categories: 1: Flat, 2: Cluster-based or Hierarchy, 3: Geography (Vahabi, 2019; Haque, 2018; Singh, 2016). This research focused on a Cluster-based routing protocol including Chain-Cluster based Mixed (CCM) routing algorithm (Marhoon, 2018; Tang, 2012), Two-Stage Chain-Based Routing Protocol (TSCP) (Kareem, 2014; Marhoon, 2018), and Deterministic Chain-Based Routing Protocol (DCBRP) (Marhoon, 2016).

Chain-cluster based mixed routing protocol:

This algorithm was proposed by Tang et al. (Marhoon, 2018; Tang, 2012). In this algorithm, the network is divided into a number of chains, each chain consisting of a number of nodes. There is a Chain Head $(\mathrm{CH})$ in each chain. Each resource node in each chain sends the data to the next node until the data is given to the $\mathrm{CH}$ node. In this network, there is a node in the middle of the network called Cluster Head and CH nodes directly deliver data to this node. The sink node also is placed next to the network. Cluster Head node collects data from the network and delivers it to the sink node.

Two-stage Chain-based routing protocol:

This protocol has been proposed by Kareem et al. (Marhoon, 2018; Kareem, 2014) and is similar to the Chaincluster based routing protocol, except that there is no Sink node in the network, the data is delivered to Cluster Head node, and the delay observed in the Chain-cluster based routing protocol does not exist in this protocol. 


\section{Deterministic Chain- Based Routing Protocol:}

This protocol has been proposed by Marhoon et al. (Marhoon, 2016) in which each three sensor nodes in the network form a cluster. The middle node in each cluster is chosen as the Cluster Head node and the other two nodes deliver their data to the Cluster Head node. Cluster Head nodes deliver the received data to the next Cluster Head node until the data is delivered to the Sink node in the network. In this protocol, the Sink node is located at the end of the network and therefore, the location of the Sink node is the main problem of this method, which causes energy loss in the network.

One of the problems with chain-based methods is the data transfer between $\mathrm{CH}$ nodes, which causes energy loss. In the newly proposed method, this stage of data transfer has been removed. One type of wireless sensor node is the gateway node, which is actually a normal wireless node but is rechargeable by solar energy and has a much lower cost than sink nodes (Nadeem, 2013; Kumar, 2018). In the proposed algorithm, instead of $\mathrm{CH}$ nodes, gateway nodes are used in each chain, which results in greater energy efficiency in the nodes and in turn, increases the lifespan of the networks. Moreover, gateway nodes are not used in chain-based routing, but rather they are used in a new way so that their algorithms are used near dangerous locations to measure faults, define lava from volcanos, etc. However, since these networks transfer data through chains, more energy is consumed by nodes that must be controlled through a better network design. Therefore, chain-based methods have yet to be completely successful. Given the problems and issues in previous methods, this research followed the goals such as improving routing in chain-based methods, lowering energy consumption in network nodes, increasing the lifespan of networks, and reducing stages of data transfer, which is directly related to energy consumption in network nodes.

\section{Proposed algorithm}

\section{Research method}

In the new method solar rechargeable gateway nodes were used, which have a much lower cost than Sink nodes (Nadeem, 2013; Kumar, 2018) and are greatly more efficient than $\mathrm{CH}$ nodes, which are the same as normal sensor nodes. The proposed model had the following features:

- Solar rechargeable nodes

- Sensor nodes are aware of their own location.

- The location of nodes is chosen by Global Routing System (GRS) or radio signals.

- Sensor nodes are aware of the location of their neighboring nodes in the chain

\section{Network model}

The sensor ground is rectangular and the distance between sensor nodes is 10 meters (Marhoon, 2016). The sensor ground is divided into a number of chains and there is a gateway node in the middle of each chain as the collector node. The total number of chains were calculated through Equation (1):

$$
N_{\text {chain }}=\left\lceil\frac{N}{N_{\text {col }}}\right\rceil
$$

In Equation (1), $N$ shows the number of sensor nodes in the network, $N_{c o l}$ shows the number of columns in the network, and $N_{\text {chain }}$ shows the total number of chains in the network. For example, if there are 50 nodes in the network and the formation of nodes is in 5 columns, there would be 10 sensor nodes in each chain. Also, the number of gateway nodes equaled the number of chains in the network, because there was a rechargeable gateway node in the middle of each chain. Therefore, Equation (2) was true:

$$
N_{\text {gateway }}=N_{\text {chain }}
$$

\section{Data delivery in the new proposed algorithm}

In each chain, when a node wants to send its data, first it sends it to its neighboring node, and the neighboring node sends it to its own neighboring node, and this process continues until the data is delivered to the collector gateway node in the chain. In the newly proposed method, each rechargeable gateway node directly sends the data to the Sink node or the BS that leads to consuming much less energy, longer network lifespan, and significantly decreased the delay in the data transfer. Figure (1) shows the network model in the new method and Figure (2) shows the overall process of the proposed algorithm. 


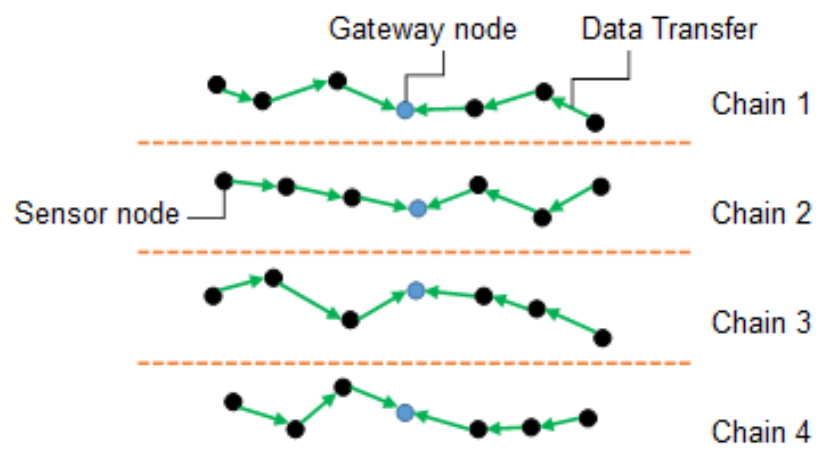

Figure (1). The network model in the newly proposed method

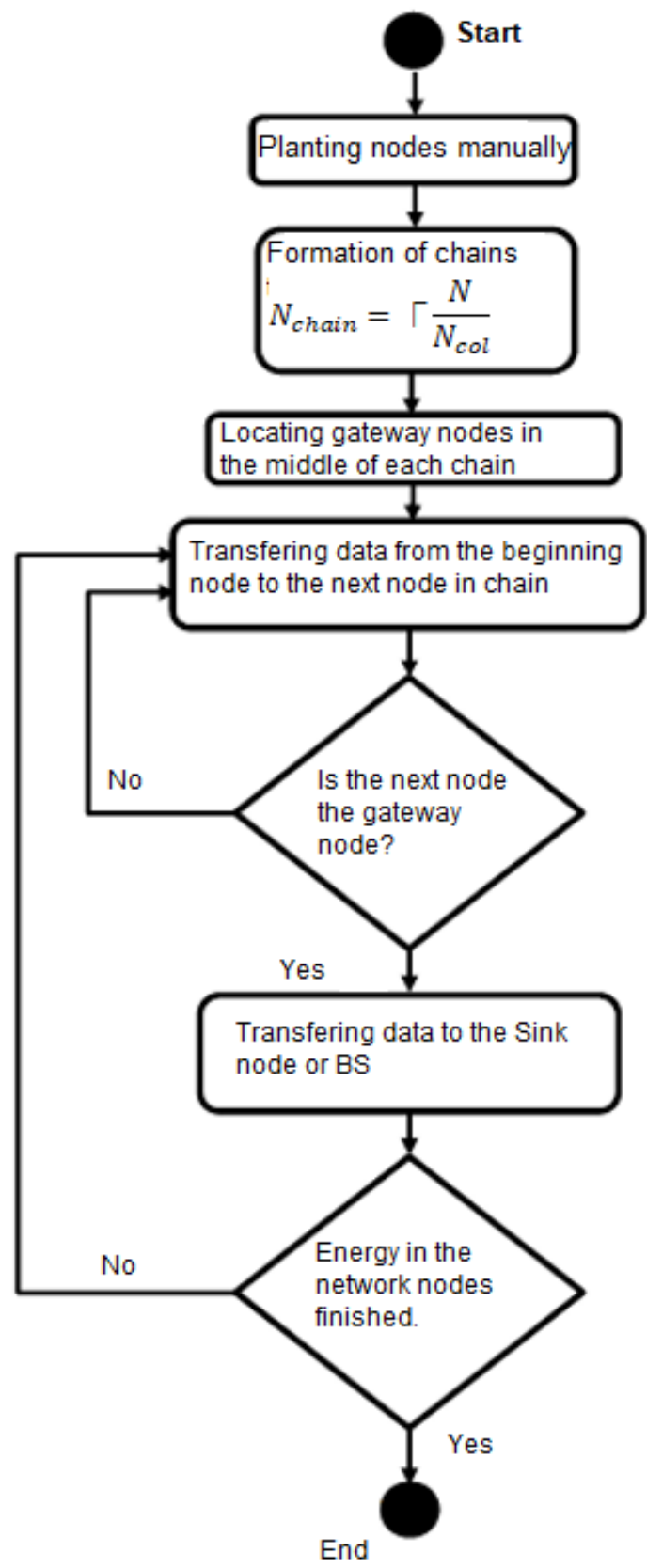

Figure (2). The overall process of the proposed algorithm 


\section{Simulations' results}

The simulator NS3 v.3.26 was used in this research (Vahabi, 2019) and the simulation was done with a total number of 100 nodes (Marhoon, 2016; Kareem, 2014). MAC layer of the network was considered IEEE 802.11 (Eslaminejad, 2012; Vahabi, 2019), which was related to wireless networks. Also, the following energy model was used for the simulation (Marhoon, 2016; Kareem, 2014). The required energy to transfer a data package of $l$ bits for the distance of $d$ meters (the distance between the starting node and the last node) was calculated using the Equation (3). Equation (4) was used to determine the energy required to receive a data package of $l$ bits. The starting energy for all nodes in all of the methods was 0.5 Joules (Ramluckun, 2017). The distance between the nodes was 10 meters (Marhoon, 2016). If a node had $1 \%$ energy remaining, it would be considered a dead node (Vahabi, 2019; Eslaminejad, 2012). Also in this simulation, the size of each data package was either 4000 bits or 500 bytes (Vahabi, 2019). The evaluation factors of these methods were specific to chain-based routing methods (Marhoon, 2016). In order to evaluate the methods in all circumstances, the simulations were run 10 times (Ghugar, 2018).

$$
\begin{aligned}
& E_{T x}(l, d)=l E_{\text {elec }}+E_{\text {amp }} * d^{2} \\
& E_{R_{x}}(l)=l E_{\text {elec }}
\end{aligned}
$$

\section{Average}

Delay is a very important factor in chain-based routing methods because the data transfer rate in this routing category is of great importance.

\section{The average delay for 300 rounds of data transfer}

Equation (5) shows the value of this parameter. In this equation, $S$ represents the number of rounds of data transfer (300 rounds). $T_{R x}$ represents the time of the package delivery, and $T_{T x}$ shows the time of sending the package.

$$
D_{\text {Average }}=\sum_{S=1}^{\text {Last } S} T_{R x}-T_{T x} / \text { Number of packets }
$$

Chart (1) shows the results of the simulation of this evaluation factor for all methods. According to Figure (1), the proposed algorithm had the lowest delay in sending the data during all rounds. Given that in the proposed algorithm, the gateway nodes were used in each chain, which were rechargeable by solar energy, so its energy could be considered unlimited. In addition, since these nodes delivered the data directly to the Sink node or the BS, they had the lowest data transfer delay.

\section{Average end-to-end delay}

This parameter shows the delay from the beginning of work in the network until it is done, and its value was calculated through Equation (6). In which, $S$ represents the rounds of work in the network from the first to the last and $D_{\text {Average }}$ represents the delay in 300 rounds of data transfer, the value of which was calculated through the Equation (5).

$$
D_{\text {Average }_{\text {EndToEnd }}}=\sum_{S=0}^{\text {Last } S} \frac{D_{\text {Average }}}{\text { Last } S \text { which Last Node Will Die }}
$$

Since the average delay directly affects the average end-to-end delay and the proposed algorithm has had the best function in that area, this factor also had the best function.

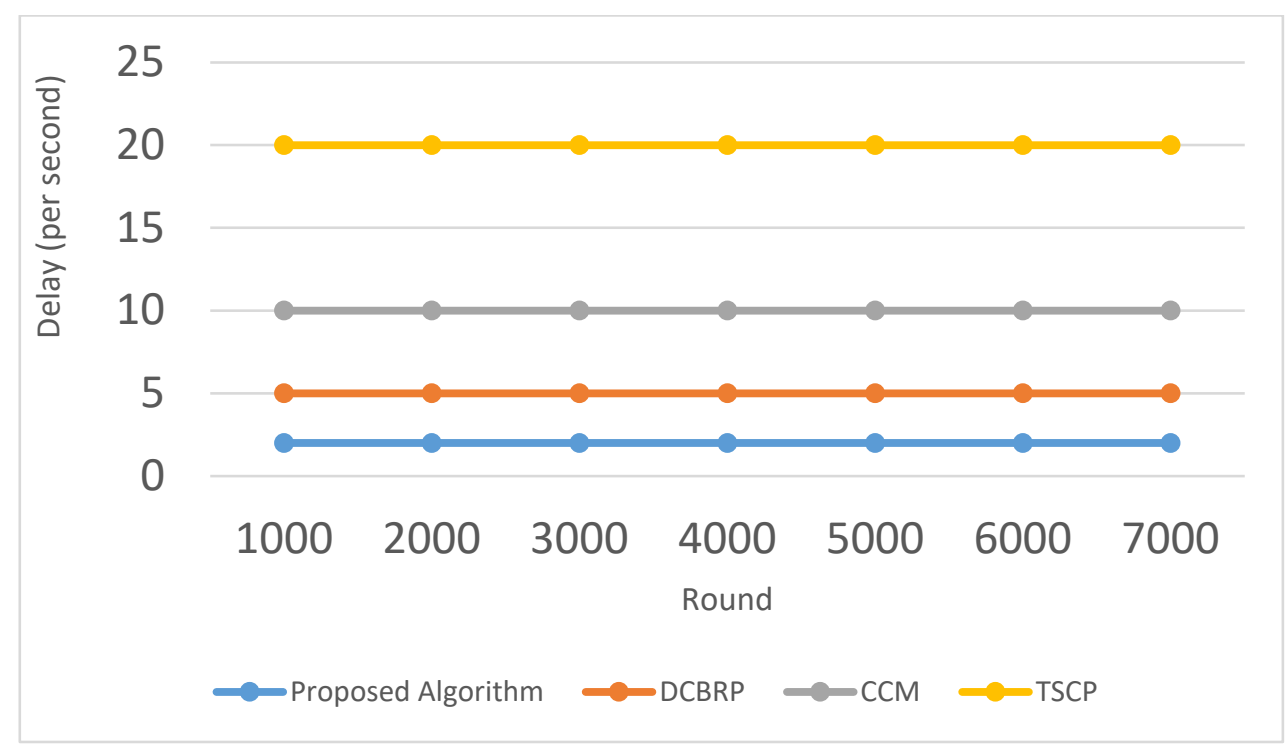

Chart (1). The average delay in each 300 rounds 


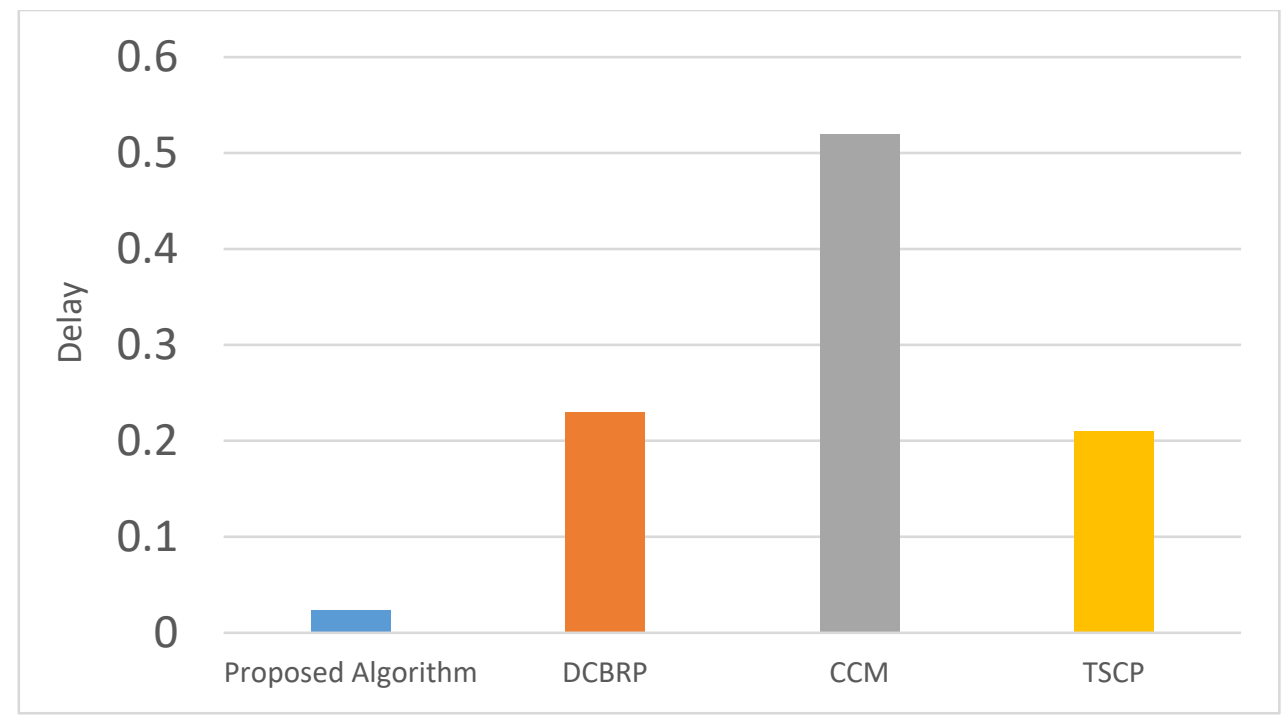

Chart (2). The average end-to-end delay

\section{Networks' lifespan}

Networks' lifespan in chain-based routing methods consists of different stages:

- When the first node dies in the network

- When half the nodes in the network die

- When all the nodes die and the work of networks is finished

Chart (3) shows the networks' lifespan in all methods. According to the chart, the proposed algorithm had the longest network lifespan, because the rechargeable gateway nodes in each chain consumed much less energy. On average, in all stages of the proposed algorithm, there was $42.46 \%, 57.97 \%$, and $65.14 \%$ longer lifespan in DCBRP, CCM, and TSCP, respectively. Chart (4) shows the Standard Deviation in average lifespan in different methods. The proposed algorithm had higher dispersion than the average lifespan of the networks and therefore, the higher deviation was proof of longer lifespan of networks in the new method. The standard deviation provided more precise details to be discussed.

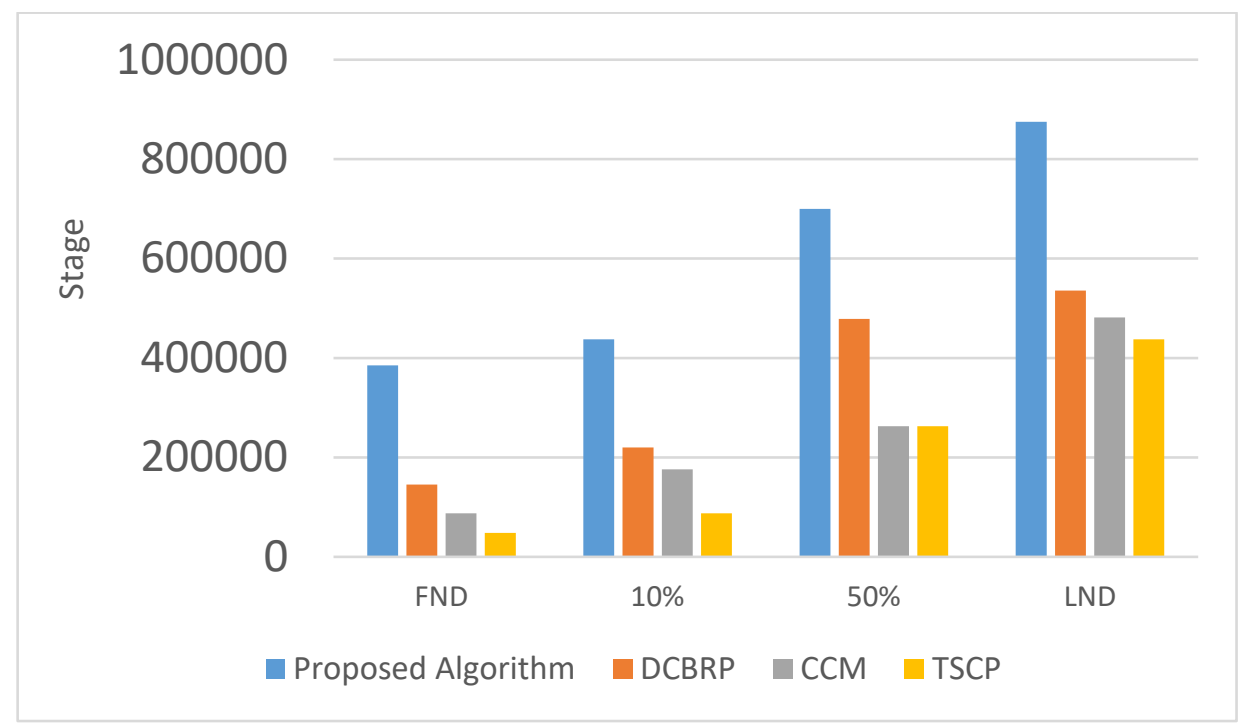

Chart (3). Average networks lifespan 


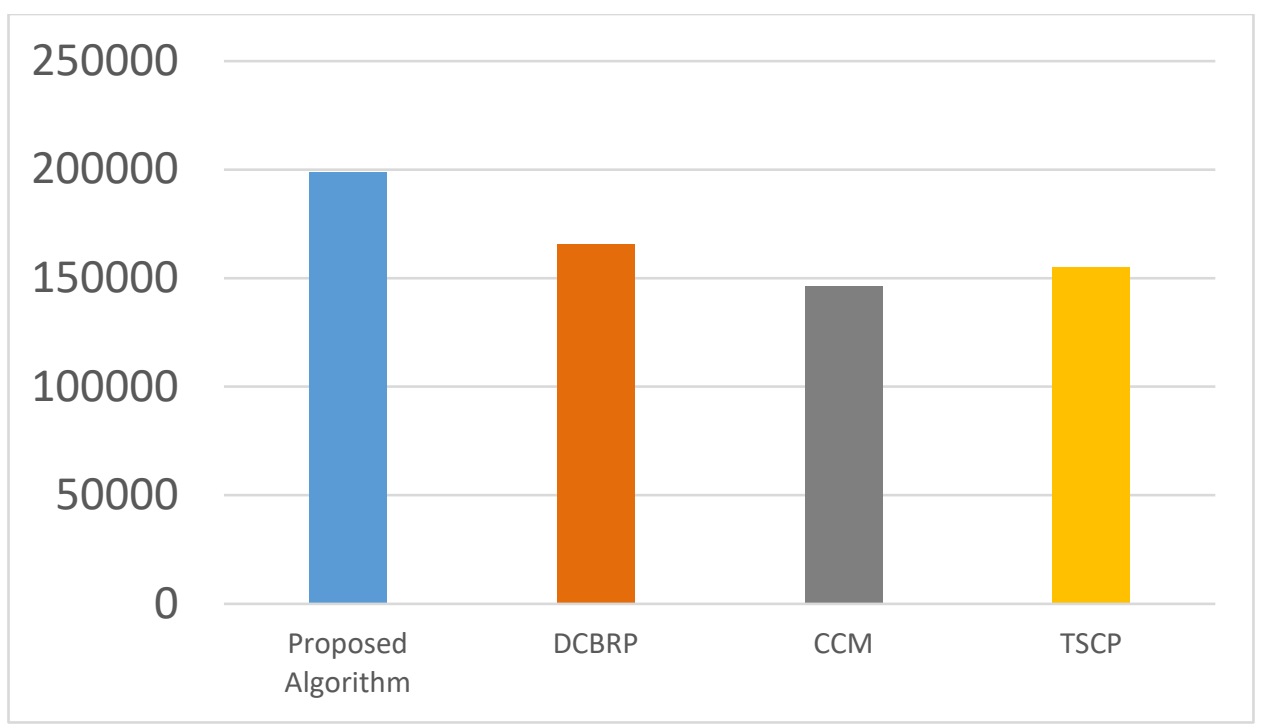

Chart (4). Standard Deviation in average networks lifespan

\section{Average networks energy consumption}

Chart (5) shows the energy consumed in the network per 300 rounds of data transfer. The proposed algorithm could conserve more energy and prolonged network lifespan and also caused other sensor nodes in the network to die later due to using rechargeable gateway nodes in each chain, which directly sent the data to BS without sending it to other gateway nodes. The proposed algorithm had reduced energy consumption by $50 \%, 75 \%$, and, $91.67 \%$ in DCBRP, CCM, and TSCP, respectively. Chart (6) shows the standard deviation in the average energy consumption in the network.

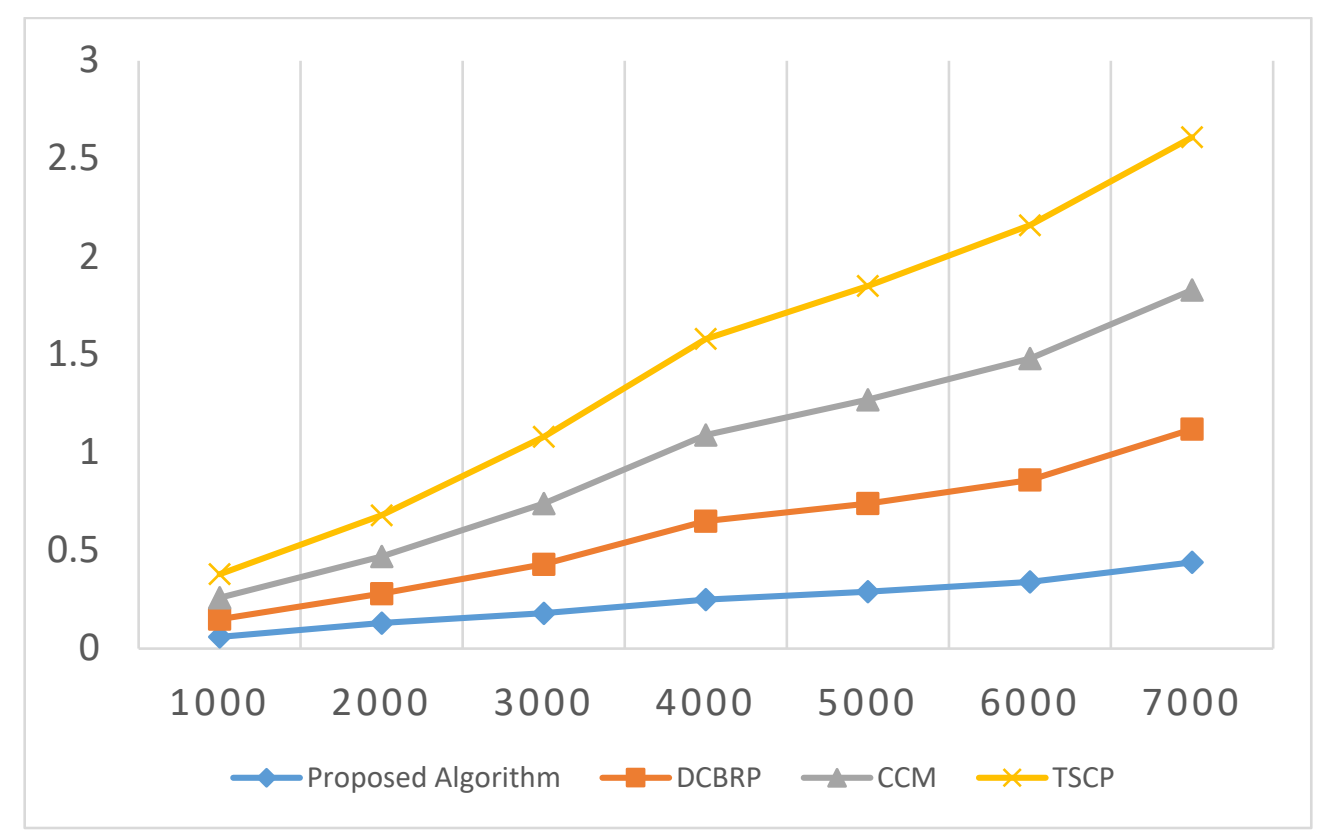

Chart (5). The average energy consumption in the network 


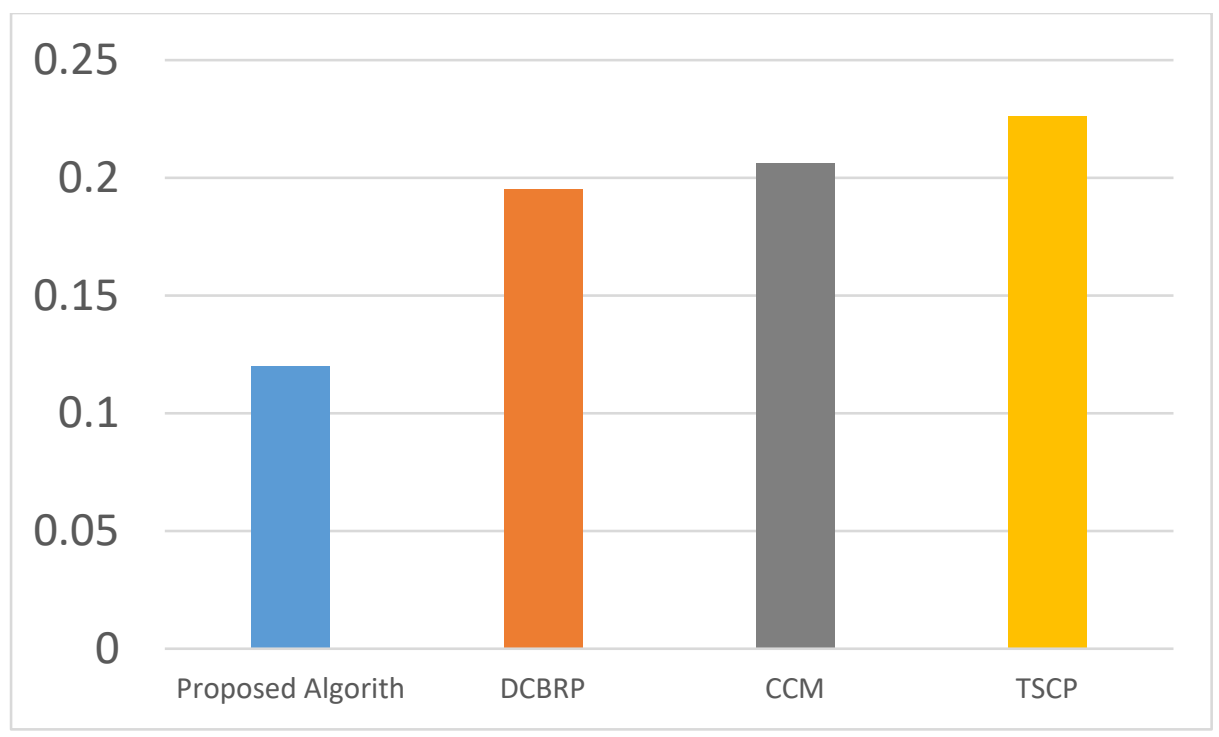

Chart (6). Standard deviation in average energy consumption in the network

\section{Consumed energy * Delay}

According to Chart (7), the proposed algorithm had the most efficient plant design and network nodes topology, because it had the lowest consumed energy * delay compared to other methods. In the new method, using solar rechargeable gateway nodes significantly reduced energy consumption by nodes, prolonged the network lifespan, reduced data transfer delay, and also reduced consumed energy * delay.

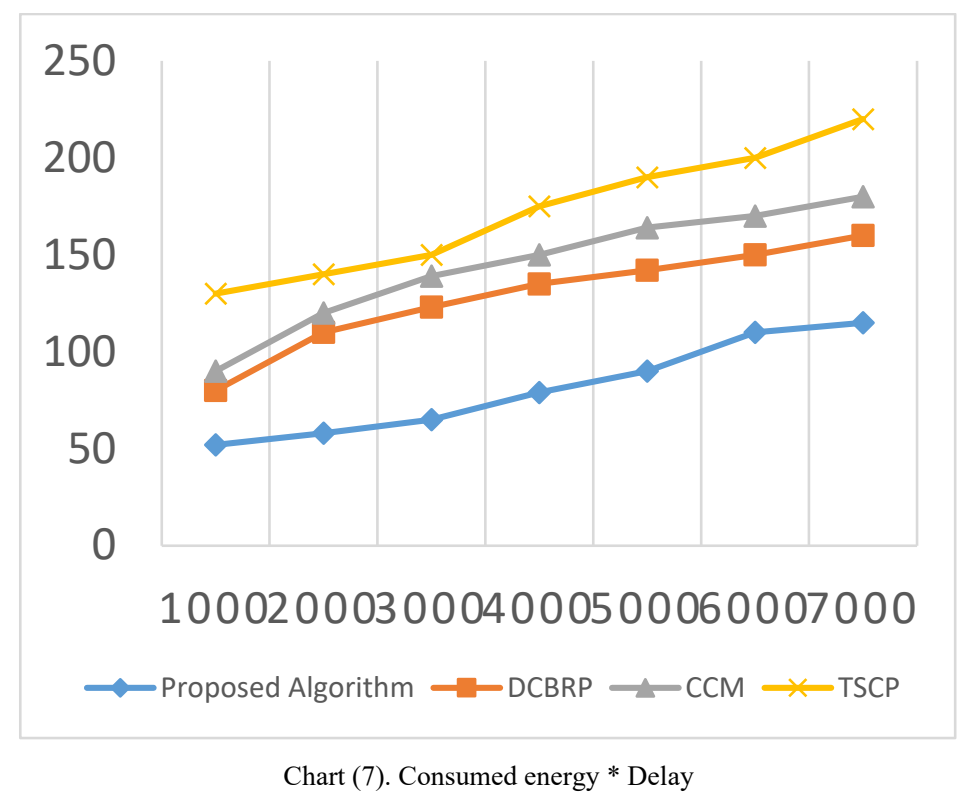

\section{Discussion and Conclusion}

The most important challenge in WSN is the energy consumption during data transfer and receiving data package, and therefore, routing data packages in this type of networks is of great importance. By designing routing that is efficient in energy consumption, the consumed energy by nodes and the network could be reduced, and also the lifespan of the networks could be prolonged. This research focused on chain-based routing and proposed a new method in this type of routing where gateway nodes were used instead of using $\mathrm{CH}$ nodes, which quickly lose their energy. A gateway node is the same as normal sensor node but it is rechargeable by solar energy, which is somewhat stable and considered unlimited. In addition, in this research, simulations were run using the NS3 software V. 3.26, because this simulator is the best tool in the field of networks and its results are closer to real-life results. The simulation results superlatively showed that in the proposed algorithm, there was at least $46.42 \%$ longer lifespan compared to other methods; as well as reduced energy consumption by at least $50 \%$ in comparison to other methods. The new algorithm is able to reduce end-to-end delay and network delay more than previous methods in chain-based routing. 
Further, in order to raise the networks' scalability in future investigations, the new method could be applied in numerous areas or regions in a way that these regions can share their information with each other to provide more precise and complete results for the final user. An efficient way of information sharing should be found in order to have more efficiency in the network.

\section{References}

[1] Akyildiz, I. F., Su, W., Sankarasubramaniam, Y., Cayirci. (2002). Wireless sensor networks: a survey. Computer Networks, 38, 393422 .

[2] Eslaminejad, M., Ismail, A. S., Razak, S. A. (2012). EEDARS: An Energy-Efficient Dual-Sink Algorithm with Role Switching Mechanism for Event-Driven Wireless Sensor Networks. KSII TRANSACTIONS ON INTERNET AND INFORMATION SYSTEMS, 6, doi:10.3837/tiis.2012.10.002.

[3] Ghugar, U., Pradhan, J., Bhoi, S. K., Sahoo, R. R., Panda, S. K. (2018). PL-IDS: physical layer trust based intrusion detection system for wireless sensor networks. Int. j. inf. tecnol.

[4] Haque, M., Ahmad, T., Imran, M. (2018). Review of Hierarchical Routing Protocols. Intelligent Communication and Computational Technologies, 237-246, doi:10.1007/978-981-10-5523-2 22

[5] Kamal, Z., Salahuddin, M. A. (2015). Introduction toWireless Sensor Networks. Wireless Sensor and Mobile Ad-Hoc Networks, 3-32.

[6] Kareem, H., Hashim, S. J., Subermaniam, S., Sali, A. (2014) ENERGY EFFICIENT TWO STAGE CHAIN ROUTING PROTOCOL (TSCP) FOR WIRELESS SENSOR NETWORKS. Journal of Theoretical and Applied Information Technology, (2)59.

[7] Kumar, Sh. (2018). Energy Aware Multi-Hop Routing Protocol for WSNs Using Gateway. International Research Journal of Engineering and Technology (IRJET).

[8] Marhoon, H. A., Mahmuddin, M., Nor, S. A. (2016). DCBRP: a deterministic chain-based routing protocol for wireless sensor networks. SpringerPlus.

[9] Marhoon, H. A., Mahmuddin, M., Nor, S. A., Hussein, M. H., Gifal, A. S. (2018). Performance evaluation of CCM and TSCP routing protocols within/without data fusing in WSNs. Renewable Energy and its Applications, doi :10.1088/1742-6596/1032/1/012054.

[10] Nadeem, Q., Rasheed, M. B., Javaid, N., Khan, Z. A., Maqsood, Y., Din, A. (2013). M-GEAR: Gateway-Based Energy-Aware MultiHop Routing Protocol for WSNs. Eighth International Conference on Broadband, Wireless Computing, Communication and Applications.

[11] Ramluckun, N., Bassoo, V. (2017). Energy-efficient chain-cluster based intelligent routing technique for Wireless Sensor Networks. Applied Computing and Informatics, doi: 10.1016/j.aci.2018.02.004.

[12] Singh, H., Singh, D. (2016). Taxonomy of Routing Protocols in Wireless: A Survey. Contemporary Computing and Informatics (IC3I), 2016 2nd International Conference. Noida, India.

[13] Tang, F., You, I., Guo, S., Guo, M., Ma, Y. (2012). A chain-cluster based routing algorithm for wireless sensor networks. J Intell Manuf, doi: 10.1007/s10845-010-0413-4.

[14] Vahabi, S., Eslaminejad, M., Dashti, S.E. (2019). Integration of geographic and hierarchical routing protocols for energy saving in wireless sensor networks with mobile sink, Wireless Netw, (25)5, 2953-2961, doi: 10.1007/s11276-019-02015-5.

[15] Warrier, M. M., Kumar, A. (2016). Energy Efficient Routing In Wireless Sensor: A Survey. IEEE WiSPNET 2016 conference. Chennai, India. 